\title{
HERMES AND THE SPIN OF THE PROTON
}

\author{
H. E. Jackson \\ Physics Division, Argonne National Laboratory, \\ Argonne, Illinois 60439, United States
}

November 2, 2018

\begin{abstract}
HERMES is a second generation experiment to study the spin structure of the nucleon, in which measurements of the spin dependent properties of semi-inclusive deep-inelastic lepton scattering are emphasized. Data have been accumulated for semi-inclusive pion, kaon, and proton double-spin asymmetries, as well as for high- $p_{T}$ hadron pairs, and singlespin azimuthal asymmetries for pion electroproduction and deep virtual Compton scattering. These results provide information on the flavor decomposition of the polarized quark distributions in the nucleon and a first glimpse of the gluon polarization, while the observation of the azimuthal asymmetries show promise for probing the tensor spin of the nucleon and isolating the total angular momentum carried by the quarks.
\end{abstract}

\section{Introduction}

As Sir Isaac Newton speculated centuries ago in his treatise, Optics, "There are therefore agents in nature able to make the particles of bodies stick together by very strong attractions. And it is the business of experimental philosophy to find them out." Indeed, one of the central challenges of contempory nuclear physics is achieving an understanding of how quarks and gluons combine to make mesons, baryons, and nuclei. One particular issue which has received much attention is how the constituent-parton spins combine to form the spin of the proton. This is not a new problem. The first experiments to explore the spin structure of the nucleon were performed 30 years ago. 11 They took the form of measurements of inclusive spin asymmetries, the basic measurement which, until now, has provided most of our knowledge of nucleon spin structure.

The objective of these studies was to determine what fraction of the spin of the nucleon was carried by the quarks. The nucleon spin can be decomposed conceptually, into the spin of its constituents according to the equation

$$
\left\langle s^{N}{ }_{z}\right\rangle=\frac{1}{2}=\frac{1}{2} \Delta \Sigma+\Delta G+\Delta L_{z}
$$


where the three terms are the quark and gluon spins, and the total orbital angular momenta of the quarks and gluons, respectively. The original expectation, based on the constituent quark model was that the intrinsic spin of the valence quarks provided the total spin, ie $\Delta \Sigma=1$. More realistic calculations with current quarks [2] resulted in a "canonical value" of $\Delta \Sigma \approx 2 / 3$. Then came the first experiments with polarized deep-inelastic lepton scattering at SLAC[1] and then CERN [3] which led to the conclusion that $\Delta \Sigma \approx 0.1-0.2$. The prospect that the fraction of the nucleon spin carried by the quarks was so small provoked what was called the "spin crisis".

With these indications of the complexity of the spin structure, it was quickly realized that a simple leading order(LO) analysis which did not include contributions from gluons was naive. More recent next-to-leading order(NLO) treatments provide a picture more consonant with our present understanding of QCD. The focus has been on the spin dependent structure function for the proton, $g_{1}\left(x, Q^{2}\right)$, given by

$$
g_{1}\left(x, Q^{2}\right)=\frac{\left\langle e^{2}\right\rangle}{2}\left[C_{N S} \otimes \Delta q_{N S}+C_{S} \otimes \Delta \Sigma+2 n_{f} C_{g} \otimes \Delta g\right]
$$

where $\left\langle e^{2}\right\rangle=n_{f}^{-1} \sum_{i=1}^{n^{f}} e_{i}^{2}, \otimes$ denotes convolution over $x$, and $\Delta q_{N S}$ and $\Delta \Sigma$ are the nonsinglet and singlet quark distributions, and $\Delta g$ is the polarized gluon distribution, respectively. Here $x$ is the usual Bjorken scaling variable, $Q^{2}$ is the squared four momentum transfer, and $n_{f}$ is the number of quark flavors. The coefficient functions, $C_{N S}, C_{S}$, and $C_{g}$, have been computed up to nextto-leading order [5] in $\alpha_{s}$. At NLO they as well as their associated parton distributions depend on the renormalization and factorization schemes. While the physical observables are scheme independent, parton distributions will be maximally scheme dependent, but related from scheme to scheme by well-defined relationships. In a recent NLO analysis $[6]$ of available data for $g_{1}$, the SMC group presented results for the Adler-Bardeen $(\mathrm{AB})$ scheme, in which case the first moment of $g_{1}, \Gamma_{1}\left(Q^{2}\right)$, is given by

$$
\int_{0}^{1} d x g_{1}\left(x, Q^{2}\right)=\frac{\left\langle e^{2}\right\rangle}{2}\left[C_{N S}\left(1, \alpha_{s}(t)\right) \Delta q_{N S}(1)+C_{s}\left(1, \alpha_{s}(t)\right) a_{0}\left(Q^{2}\right)\right]
$$

where we have followed the notation of Ref. [4] in denoting moments of coefficient functions and parton densities as $f(N)=\int_{0}^{1} d x x^{N-1} f(x)$. In the same scheme the singlet axial charge, $a_{0}$, is

$$
a_{0}=\Delta \Sigma\left(1, Q^{2}\right)-3 \frac{\alpha_{s}\left(Q^{2}\right)}{2 \pi} \Delta g\left(1, Q^{2}\right) .
$$

The SMC group finds that the analysis of the $Q^{2}$ evolution of the world data base gives an singlet axial charge, $a_{0}=0.23 \pm 0.07$ (stat) \pm 0.19 (stat), and a gluonic first moment, $\Delta g\left(1,1 G e V^{2}\right)=0.99_{-0.31}^{+1.17}(\text { stat })_{-0.22}^{+0.42}(s y s t)_{-0.45}^{+1.43}(t h)$. The resulting value of the singlet quark distribution is $\Delta \Sigma=0.38$. However, the large uncertainties in $\Delta g$ preclude a precise determination of $\Delta \Sigma$ in the absence 
of direct measurements of $\Delta g$ [7]. An additional issue in the analysis of the inclusive data from these experiments, is their sensitivity to $\mathrm{SU}(3)$ symmetry breaking. In a recent study, Leader and coworkers 8 find that the strange quark and gluon polarizations vary rapidly with variations in the $\mathrm{SU}(3)$ octet axial charge. Clearly, these flavor separated polarizations are strongly dependent on the assumption of $\mathrm{SU}(3)$ symmetry.

The key to further progress is more specific probes of the individual contributions of Eq. (11) to the proton spin. Determination of the polarization of the gluons is clearly of very high priority, and in addition, a more precise measurement will eliminate a major current ambiguity in the implications of existing inclusive data. A more direct determination of the strange quark polarization, $\Delta s$, will avoid the need for the use of data from hyperon decay and the assumption of SU(3) symmetry. Measurements which are sensitive to quark charges will allow the separation of quark and antiquark polarizations. The HERMES experiment attempts to achieve these objectives, by emphasizing semi-inclusive DIS in which a $\pi, K$, or $p$ is observed in coincidence with the scattered lepton. The added dimension of flavor in the final hadron provides a valuable probe of the flavor dependence and other features of polarized parton distributions. In the sections which follow, we present a brief description of the HERMES experiment followed by reports on recent results on the flavor decomposition of polarized parton distributions, gluon polarization, transverse spin physics, and deep-virtual Compton scattering. Indeed, such studies of semi-inclusive deep-inelastic scattering appear to mark a major advance in unraveling the spin structure of the proton.

\section{The HERMES Experiment}

Deep inelastic scattering events are generated in the HERMES experiment by the interaction of the polarized lepton beam of the HERA accelerator at DESY with polarized target gases which are injected into a $40 \mathrm{~cm}$ long, tubular openended storage cell [9] located at an interaction point on the lepton orbit. The lepton beam is self polarized by the Sokolov-Ternov effect 10] with a polarization time which is typically about 20 minutes. The beam polarization is measured continuously with Compton backscattering of circularly polarized laser beams 11, 12. The beam polarization is routinely about 0.55 . Spin rotators in the ring provide longitudinal polarization at the interaction point. The polarized target gases, atomic $\mathrm{H}$ or $\mathrm{D}$ are generated by an atomic-beam source based on Stern-Gerlach separation which provides an areal density of about $2 \times 10^{14}$ atoms $/ \mathrm{cm}^{2}$ for $\mathrm{H}$ and $7 \times 10^{13}$ atoms $/ \mathrm{cm}^{2}$ for $\mathrm{D}$. The nuclear polarization is measured with a Breit-Rabi polarimeter 13 and the atomic fraction with a target gas analyser 14. The target polarization is reversed within short time intervals to minimize systematic effects. The relative lumimosity is measured 15] by

detecting Bhabha- or Mott-scattered target electrons in coincidence with the scattered lepton, in a pair of $\mathrm{NaBi}\left(\mathrm{WO}_{4}\right)_{2}$ electromagnetic calorimeters.

The HERMES spectrometer [16], shown in Fig. (11) is a forward angle open 


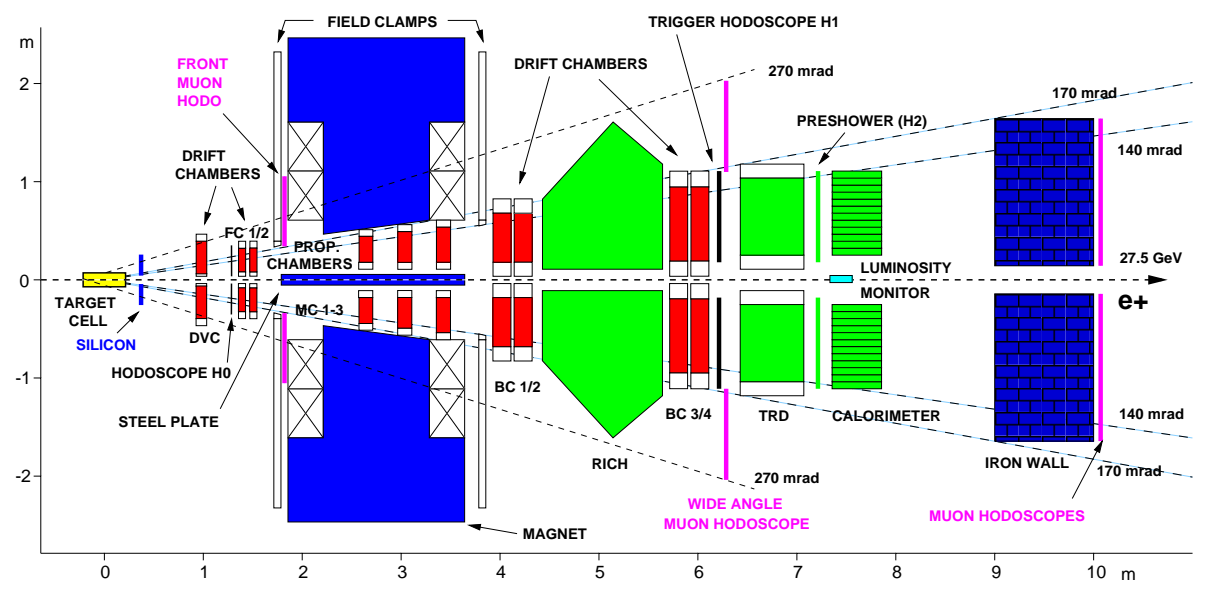

Figure 1: The HERMES Spectrometer.

geometry system consisting of two halves which are symmetric about a central horizontal shielding plate in the spectrometer magnet. A fly's eye calorimeter and a transition radiation detector furnish clean separation of hadrons and leptons. Identification of $\pi^{\prime} s, K^{\prime} s$ and $p^{\prime} s$ is accomplished by means of a novel dualradiator ring-imaging Cerenkov counter(RICH) 17 which is located between the rear tracking chambers. The combination of radiators consisting of a wall of clear aerogel and a gas volume of $C_{4} F_{10}$ provide clean particle identification over almost the full acceptance of HERMES, i.e. 2-15 GeV. The scattered leptons and hadrons produced within an angular acceptance of $\pm 170 \mathrm{mr}$ horizontally, and $40-140 \mathrm{mrad}$ vertically are detected and identified. Typical kinematics for studies of DIS are $E=27.5 \mathrm{GeV}$ for the incident lepton, $x>0.02$ where $x=Q^{2} / 2 M \nu$ is the Bjorken scaling variable, $0.1 \mathrm{GeV}^{2}<Q^{2}<15 \mathrm{GeV}^{2}$ with $-Q^{2}$ the square of the momentum transfer, and $\nu<24 \mathrm{GeV}$ where $\nu=E-E^{\prime}$ with $E\left(E^{\prime}\right)$ is the energy of the incoming(scattered) lepton in the target rest frame. To insure that hadrons detected are in the current fragmentation region, cuts of $z=E_{h} / \nu>0.2$ and $x_{F} \approx 2 p_{\text {parallel }}^{h} / W>0.1$ are imposed, where $W=\sqrt{2 M \nu+M^{2}-Q^{2}}$ is the invariant mass of the photon-proton sytem.

\section{Flavor Decomposition}

One of the principal goals from the earliest days of the HERMES experiment has been to use semi-inclusive deep-inelastic scattering to determine the separate contributions $\Delta q_{f}(x)$ of the quarks and antiquarks of flavor $f$ to the total spin of the nucleon. By means of the technique of flavor tagging, individual spin contributions can be determined directly from spin asymmetries of hadrons with the appropriate flavor content. For example, the spin asymmetry of $K^{-}$, an all sea object, will have a high sensitivity to the polarization of the quark sea. The 
measured semi-inclusive spin asymmetry, $A_{\|}^{h}$, and the corresponding photonnucleon asymmetry, $A_{1}^{h}$, for the hadron of type $h$ are given by

$$
A_{\|}^{(h)}=\frac{N_{(h)}^{\uparrow \downarrow}-N_{(h)}^{\uparrow \uparrow}}{N_{(h)}^{\uparrow \downarrow}+N_{(h)}^{\uparrow \uparrow}}, \quad A_{1}^{h}=\frac{A_{\|}^{h}}{D(1+\eta \gamma)},
$$

where, for simplicity, we assume unity beam and target polarizations and constant luminosity. Here $D$ is the depolarization factor for the virtual photon, $\eta$ is a kinematic factor [18], and $N^{\uparrow \uparrow}\left(N^{\uparrow \downarrow}\right)$ are the number of DIS events with coincident hadrons for target polarization parallel (anti-parallel) to the beam polarization. In leading order QCD assuming the validity of factorization, one can write the semi-inclusive DIS cross section, $\sigma^{h}\left(x, Q^{2}, z\right)$, to produce a hadron with energy fraction, $z=E_{h} / \nu$ as

$$
\sigma^{h}\left(x, Q^{2}, z\right) \propto \Sigma_{f} e_{f}^{2} q_{f}\left(x, Q^{2}, z\right) D_{f}^{h}\left(x, Q^{2}\right)
$$

where the sum is over quark and antiquark types $f=(u, \bar{u}, d, \bar{d}, s, \bar{s}) . \quad E_{h}$ is the energy of the hadron. The quark charge, $e_{f}$, is in units of the elementary charge. In this approximation,

$$
\begin{aligned}
A_{1}^{h}(x, z) & =\frac{\int_{z_{\min }}^{1} d z \sum_{f} e_{f}^{2} q_{f}(x) \cdot D_{f}^{h}(z)}{\int_{z_{\min }}^{1} d z \sum_{f^{\prime}} e_{f^{\prime}}^{2} q_{f^{\prime}}(x) \cdot D_{f^{\prime}}^{h}(z)} \cdot \frac{\Delta q_{f}(x)}{q_{f}(x)} \cdot \frac{1+R\left(x, Q^{2}\right)}{1+\gamma^{2}} \\
& =\sum_{f} P_{f}^{h}(x, z) \frac{\Delta q_{f}(x)}{q_{f}(x)} \cdot \frac{1+R\left(x, Q^{2}\right)}{1+\gamma^{2}} .
\end{aligned}
$$

The quantities, $P_{f}^{h}(x, z)$, are the integrated purities 19, 20] which are defined by Eq. (8). They are spin-independent quantities in leading order and represent the probability that the quark, $q_{f}$ was struck in the DIS event. The ratio $R=\sigma_{L} / \sigma_{T}$ of the longitudinal to transverse photon cross section corrects for the longitudinal component that is included in experimental parameterizations of $q_{f}\left(x, Q^{2}\right)$ but not in $\Delta q_{f}\left(x, Q^{2}\right)$. The term $\gamma=\sqrt{Q^{2}} / \nu$ is a kinematic factor. By incorporating the correction factor in the purities, one can rewrite Eq. (8) in a matrix form as

$$
A(x)=P(x) \cdot Q(x)
$$

where $A(x)$ becomes a vector whose elements are all the integrated measured asymmetries which are to be included in the analysis. The $Q(x)$ vector contains the quark and antiquark polarizations. These quantities are now connected by the purity matrix which contains the effective integrated purities. The determination of the quark polarizations from the experimentally measured spin asymmetries is reduced to the task 21] of inversion of Eq. (9) to obtain $Q(x)$.

The purity formalism has been used in the HERMES analysis to make a flavor decomposition into polarized quark distributions for $u, \bar{u}, d, \bar{d}$, and $s+\bar{s}$. For the first time, a global analysis of inclusive spin asymmetries and semiinclusive spin asymmetries for $\pi^{+}, \pi^{-}, K^{+}$, and $K^{-}$has been carried out for 

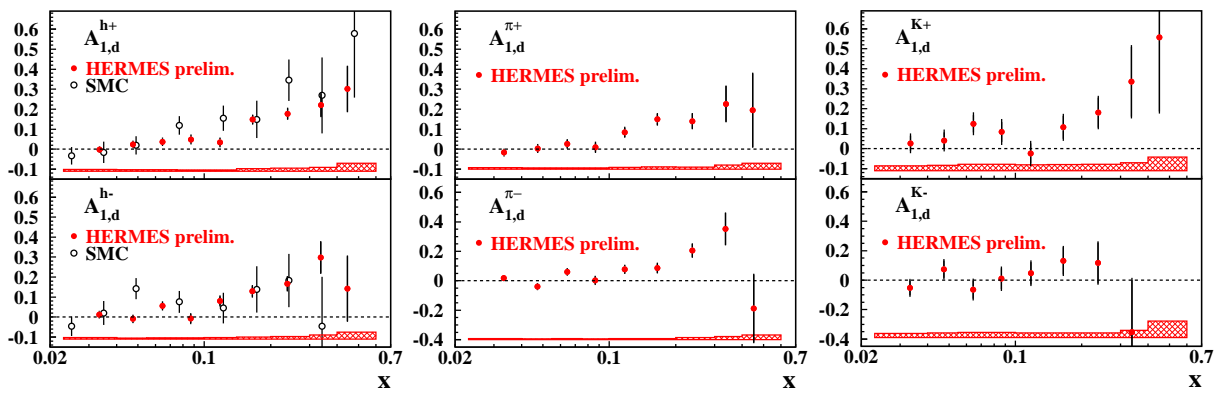

Figure 2: Semi-inclusive hadron, pion and kaon asymmetries for a deuterium target. The hadron asymmetries are compared with data from the SMC collaboration. The error bars of the HERMES data are statistical and the bands are systematic uncertainties. These data form the deuteron portion of the data base used in the purity analysis described in the text.

longitudinally polarized targets of hydrogen, and deuterium. The measured spin asymmetries $A_{1}^{h}\left(x, Q^{2}, z\right)$ were integrated in each $x$ bin over the corresponding $Q^{2}$-range and the $z$-range from 0.2 to 1 to yield $A_{1}^{h}(x)$. The data for $A_{1}^{h}(x)$ obtained with the deuterium target are shown in Fig. (2). There the semi-inclusive data are compared to earlier results from the SMC collaboration[22. The purities were obtained with a Monte Carlo calculation which used CTEQ5 leading order parton distributions 23 and a LUND fragmentation model 24 tuned to HERMES kinematics. The simulation included effects of the acceptance of the experiment. Systematic uncertainties were estimated by varying the fragmentation parameters and using alternative parton distributions 25.

The results of the decomposition obtained by solving Eq. (9) are presented in in Fig. (3). A symmetric strange sea polarization was assumed, i. e. $\Delta s / s=$ $\Delta \bar{s} / \bar{s}$. The general features of quark densities follow those of earlier decompositions 26 , 27]. The $u$-quarks show a strong positive polarization, while the $d$-quarks have a substantial negative polarization. The non-strange sea quarks are not significantly polarized. However, the strange sea appears to be positively polarized, contrary to the conclusions drawn within leading order QCD 28] from earlier inclusive data. The triplet strength $\Delta q_{3}=\Delta u-\Delta d$ extracted from the HERMES data is in agreement with the Bjorken sum rule. The polarization of the strange sea can be extracted directly from the same data set by means of a purity analysis which uses only two spin asymmetries, $A_{1}^{D}(x)$ and $A_{1, D}^{K^{+}+K^{-}}(x)$. For this analysis, fragmentation functions from $e^{+} e^{-}$collider experiments can be used to calculate purities. This method measures the quantity $\Delta s+\Delta \bar{s}$ with no assumption about strange sea symmetry, and provides an independent check of the result from the five-component decomposition. The results obtained show the same trend of positive strange sea polarization. This unexpected result poses a challenge to our understanding of the quark sea. To the extent that the sea arises from gluon splitting, one would expect from the result for the strange sea 


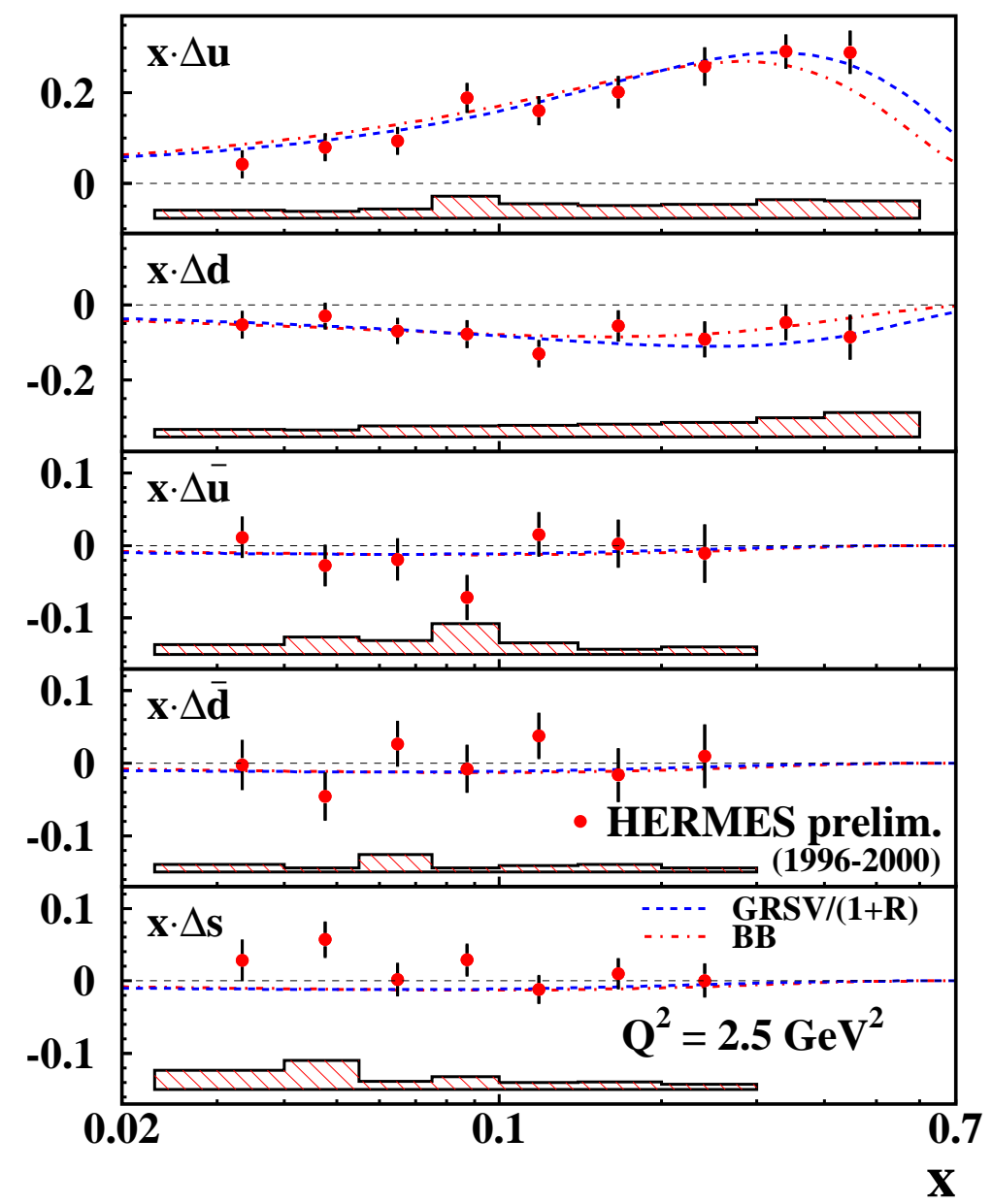

Figure 3: The $x$-weighted polarized quark densities. The plots show a five parameter fit to the data assuming a symmetric strange sea polarization. The data have been evolved to a common $Q^{2}=2.5 \mathrm{GeV}^{2}$. The dashed line shows a GRSV parameterization, and the dashed-dotted curve an alternate parameterization of Bluemlein and Boettcher (hep-ph/0203155). 
that $\Delta \bar{u} \approx \Delta \bar{d}>0$.

The data do not support recent conjectures of a strong breaking of the flavor symmetry of the light sea. The results for the quantity $\Delta u-\Delta d$ are shown in Fig. (4) together with a prediction based on the chiral quark soliton model [29] $(\chi \mathrm{QSM})$. Although the statistics are limited, the data indicate that any flavor asymmetry in the nonstrange sea is substantially smaller that the prediction of the $\chi$ QSM. In addition, the conclusion that a large negative polarization of the strange sea is the explanation for the violation of the Ellis-Jaffe sum rule in inclusive DIS is ruled out by the new results from HERMES. Analysis of the HERMES data is continuing. A point of major interest which will emerge is a determination of the octet strength $\Delta q_{8}=\Delta u+\Delta d-2 \Delta s$ as a test of $\mathrm{SU}(3)$ symmetry. The HERMES results represent the first complete flavor decomposition of the quark contribution in Eq. (1) to the spin of the nucleon.

\section{Gluon Polarization}

Indications from the analysis of the $Q^{2}$ evolution of inclusive spin asymmetries 4 , 6] suggest that the first moment of the gluon polarization, $\Delta g\left(1, Q^{2}\right)$ is positive and large, although the precise value is poorly constrained. A direct measure-

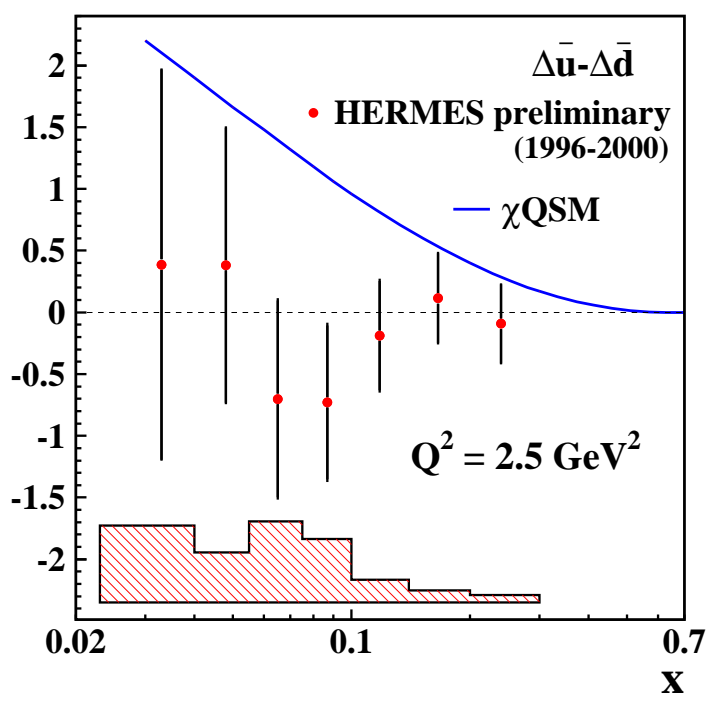

Figure 4: Flavor asymmetry $\Delta u-\Delta d$ of the light sea extracted from the HERMES five-component purity analysis. The curve describes a prediction of the $\chi$ QSM model (see text). The error bars give statistical uncertainties and the shaded band the systematic error. 

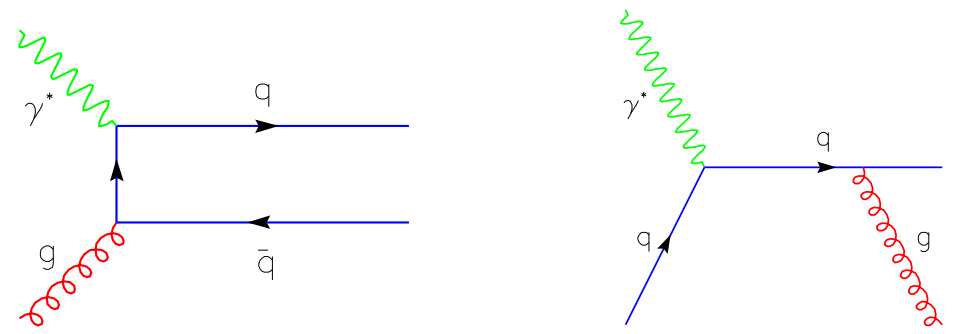

Figure 5: Feyman diagrams for processes which are major contributors to production of high- $p_{T}$ pairs, photon-gluon fusion(left) and QCD Compton scattering(right).

ment of $\Delta g\left(1, Q^{2}\right)$ is of high priority in understanding the proton spin, and much effort is focused on its measurement. A number of processes have been suggested as probes of this quantity. The photon gluon fusion process, shown in Fig. (5) is an obvious choice. Two experimental signatures of this process are charm production and dijet production with high transverse momentum, $p_{T}$. Both have been used to measure directly the unpolarized gluon structure function, $g\left(x_{g}, Q^{2}\right)$. Bravar et al. 30 have noted that at the lower energies characteristic of fixed target experiments, high- $p_{T}$ hadrons can serve as pseudo jets in a measurement of $\Delta g\left(1, Q^{2}\right)$. The spin asymmetry asymmetry associated with this process is expected to provide a large sensitivity to $\Delta g\left(x_{g}, Q^{2}\right)$.

HERMES has exploited this sensitivity by measuring the spin asymmetry in photoproduction of pairs of high- $p_{T}$ hadrons produced on a polarized hydrogen target. Events are selected by requiring at least two hadrons of opposite charge with an invariant mass assuming both hadrons to be pions of $M(2 \pi)>1.0$ $\mathrm{GeV} / \mathrm{c}^{2}$ to suppress contributions from vector mesons. The observation of the scattered lepton is not required in the event, thus allowing inclusion of the near real photoproduction region $\left(Q^{2} \approx 0\right)$ which dominates the measured cross section. Fig. (6) presents $A_{\|}$as measured for the highest $p_{T}$ values accessible in HERMES. Here events are selected if they contain hadron pairs of opposite charge, each with $p>4.5 \mathrm{GeV} / \mathrm{c}$, and a transverse momentum $p_{T}>0.5 \mathrm{GeV} / \mathrm{c}$ where $p_{T}$ is defined as the momentum transverse to the incident beam. In the top(bottom) panel the positive(negative) hadron was required to have a $p_{T}>1.5 \mathrm{GeV} / \mathrm{c}$ and $A_{\|}$is plotted as a function of the $p_{T}$ of the opposite charge. For the case where the negative hadron has $p_{T}>1.5 \mathrm{GeV} / \mathrm{c}$, a substantial negative asymmetry is observed when $p_{T}>1.0 \mathrm{GeV} / \mathrm{c}$ for the positive hadron. This negative asymmetry is to be contrasted with the positive asymmetries expected from DIS on protons, or the small positive asymmetries associated with diffractive production of vector mesons. Combining the data of the two panels of Fig. (6) over the the bins where $p_{T}^{h_{1}}>1.5 \mathrm{GeV} / \mathrm{c}$ and $p_{T}^{h_{2}}>1.0 \mathrm{GeV} / \mathrm{c}$ where $h_{1}$ signifies the hadron with the higher $p_{T}$ yields a negative asymmetry $A_{\|}=-0.28 \pm 0.12$ (stat.) \pm 0.02 (syst). When the requirement $p_{T}^{h_{1}}>1.5 \mathrm{GeV} / \mathrm{c}$ 

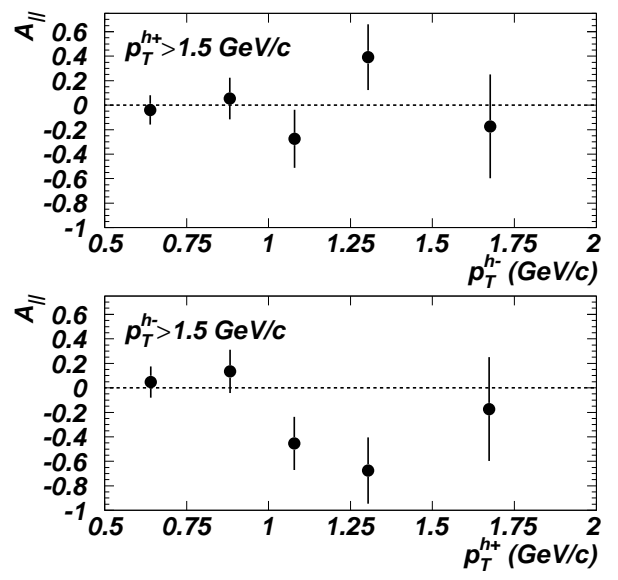

Figure 6: $A_{\|}\left(p_{T}^{h^{+}}, p_{T}^{h^{-}}\right)$for $p_{T}^{h^{+}}>1.5 \mathrm{GeV} / \mathrm{c}$ (top) and for $p_{T}^{h^{-}}>1.5 \mathrm{GeV}$ (bottom). The error bars represent the statistical uncertainty.

is relaxed, $A_{\|}$is consistent with zero.

While a number of processes can, in principle, contribute the asymmetry for high- $p_{T}$ hadron pairs, Monte-Carlo simulations using LEPTO and PYTHIA event generators 31. show that only photon-gluon fusion (PGF) and the QCD Compton effect (QCDC) generate significant asymmetries at the kinematics of Fig. (6). In this case the experimental asymmetry takes the form

$$
A_{\|}=\left(A_{P G F} f_{P G F}+A_{Q C D C} f_{Q C D C}\right) D,
$$

where $f_{i}$ is the unpolarized fraction of events from process $i$ and $D$ is the virtual photon depolarization parameter. In the HERMES analysis, the asymmetries $A_{i}^{\prime} s$ have been approximated by products of hard-process asymmetries and parton polarizations. These hard-process asymmetries $\hat{a}_{P D F}=\hat{a}(\gamma g \rightarrow q \bar{q})$ and $\hat{a}_{Q C D C}=\hat{a}(\gamma g \rightarrow q g)$ are calculable in (LO) QCD 32]. The results are -1 and $\approx 0.5$ respectively for the kinematics of this measurement and independent of flavor. The measured asymmetry can then be expressed in terms of the total quark polarization $\Delta q / q$ and the gluon polarization $\Delta G / G$ as

$$
A_{\|} \approx\left(\hat{a}_{P G F} \frac{\Delta G}{G} f_{P G F}+\hat{a}_{Q C D C} \frac{\Delta q}{q} f_{Q C D C}\right) D
$$

where the kinematic dependences have been suppressed. Eq. (11) was used to extract values of $\Delta G / G$ for each of the four values of $A_{\|}$measured at $p_{T}^{h^{2}}>0.8 \mathrm{GeV} / \mathrm{c}$ which are obtained by averaging the corresponding asymmetries shown in Fig. (6). The resulting values were then averaged to obtain the value $\Delta G / G=0.41 \pm 0.18($ stat $) \pm 0.03$ (syst) for $\left\langle x_{G}\right\rangle=0.17$ and $\left\langle\hat{p}_{T}^{2}\right\rangle=2.1$ $(\mathrm{GeV} / \mathrm{c})^{2}$. This value of $\Delta G / G$ is compared with a number of phenomenological LO QCD fits to world data on $g_{1}\left(x, Q^{2}\right)[33$, 34 in Fig. (7). The horizontal error 


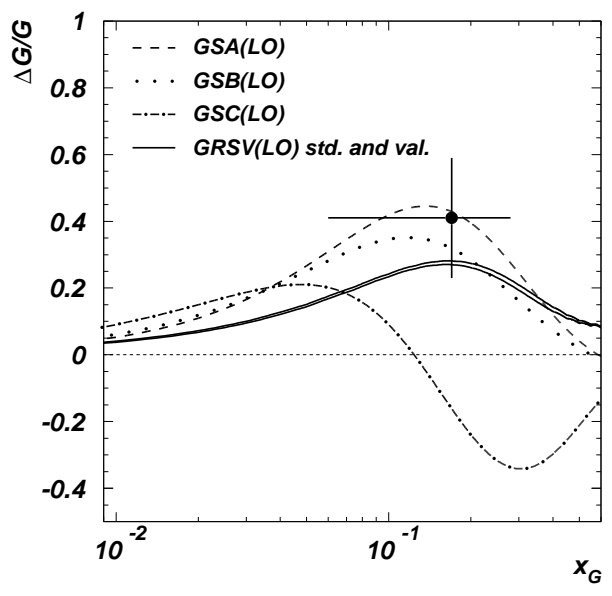

Figure 7: The extracted value of $\Delta G / G$ compared with phenomenological QCD fits to a subset of the world's data on $g_{1}^{p, n}\left(x, Q^{2}\right)$. The curves are from Ref's. 33,34 evaluated at a scale of $2(\mathrm{GeV} / \mathrm{c})^{2}$. The error indicated on $\Delta G / G$ represents statistical and experimental systematic uncertainties only; no theoretical uncertainty is included.

bar represents the standard deviation of the $x_{G}$ distribution which corresponds to the kinematic range for the hadrons measured, as given in the Monte Carlo simulation. Measurements have recently been extended to the study of high- $p_{T}$ kaon pairs for which the sensitivity to $\Delta G / G$ is enhanced by the suppression of fragmentation of non-strange quarks into strange hadrons. As was the case for unidentified hadron pairs, for kaon pairs negative spin asymmetries are observed when both kaons have $p_{T}>1.0 \mathrm{GeV} / \mathrm{c}$. Again the data suggest a substantial positive gluon polarization.

\section{Transverse Spin Physics}

Three structure functions are required to provide a complete description of the quark structure of the proton at leading order. They are the unpolarized structure function, $f_{1}\left(x, Q^{2}\right)$, the longitudinal spin structure function, $g_{1}\left(x, Q^{2}\right)$, and a transverse structure function (transversity), $h_{1}\left(x, Q^{2}\right)$ which measures the quark spin distribution perpendicular to its momentum at infinite momentum. Because $h_{1}\left(x, Q^{2}\right)$ is chiral odd, it is not measurable in inclusive DIS. However, it is of considerable intrinsic interest because of its unique properties. At low scales, $Q^{2} \approx 1 \mathrm{GeV}^{2}$, most theories give $h_{1}\left(x, Q^{2}\right) \approx g_{1}\left(x, Q^{2}\right)$, while the first moment of $h_{1}\left(x, Q^{2}\right)$, the "tensor charge" includes only valence contributions. The $Q^{2}$ evolution of $h_{1}\left(x, Q^{2}\right)$ is much simplier than that of its LO brothers, because it does not couple to gluons. It is very much a valence quantity. Transversity can be probed 35] by measuring the azimuthal distribu- 


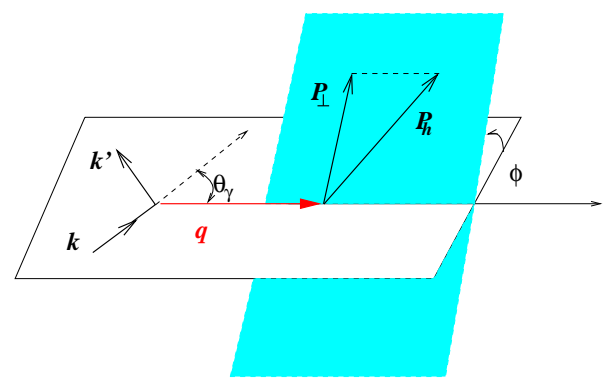

Figure 8: Kinematics for pion electroproduction in semi-inclusive DIS.

tion of hadrons produced in polarized DIS, and with highest sensitivity from a transversely polarized target.

In spite of its odd chirality, $h_{1}\left(x, Q^{2}\right)$ is accessible in semi-inclusive DIS if combined with a fragmentation process which also has a chiral odd structure. This chiral odd structure results from the Collins effect [36], i.e. a correlation between the axis of transverse spin and $\vec{p}_{\pi} \times \vec{q}$, a chiral odd correlation. Single spin asymmetries are well known in proton-induced pion production from the data of FNAL experiment E704 37] at $200 \mathrm{GeV}$. More recent theoretical calculations by Mulders and Tangerman [38] have established, at LO, that $\sin (\phi)$ and $\sin (2 \phi)$ azimuthal variations in pion single spin asymmetries signal chiral-odd fragmentation. This establishes the very exciting possibility that spin asymmetries such as $A_{p \uparrow}^{\sin (\phi)}\left(p_{t}\right)>0$ can be used as quark polarimeters for measurements of transversity in the proton.

HERMES has made the first measurements of single-spin azimuthal asymmetries for semi-inclusive pion production in DIS, using both unpolarized and longitudinally polarized proton targets in the HERA $27.5 \mathrm{GeV}$ polarized positron storage ring. The kinematic cuts for these measurements were $1 \mathrm{GeV}^{2}<Q^{2}<$ $15 \mathrm{GeV}^{2}, W>2 \mathrm{GeV}, 0.023<x<0.4$ and $y<0.85$. Pions were identified over the range $4.5 \mathrm{GeV}<E_{\pi}<13.5 \mathrm{GeV}$. Exclusive production was suppressed with the requirement $0.2<z<0.7$. The limit $p_{\perp}>50 \mathrm{MeV}$ was applied to the pions to insure accurate measurement of the angle $\phi$. Measurements were made with all combinations of beam and target helicities to permit measurement of single and double spin asymmetries in the cross section. The kinematics of pion electroproduction are presented in Fig. (8). Here $k$ and $k^{\prime}$ are the four momenta of the incoming and outgoing lepton, respectively. The transverse momentum $\left(p_{\perp}\right)$ of the pion is defined with respect to the virtual photon direction in the initial photon-proton center-of-mass system. The angle $\phi$ is the Collins angle which provides the chiral odd azimuthal dependence in the fragmentation process. The HERMES measurements of the single spin asymmetry

$$
A_{U L}(\phi)=\frac{1}{P} \frac{N^{+}(\phi)-N^{-}(\phi)}{N^{+}(\phi)+N^{-}(\phi)}
$$



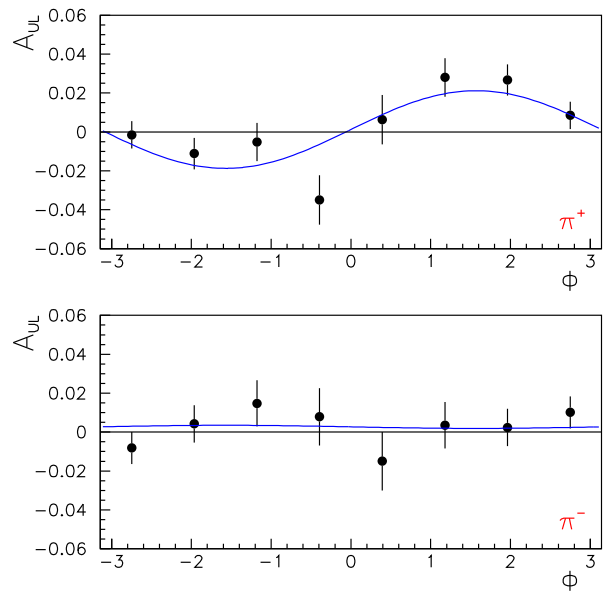

Figure 9: Single spin asymmetry $A_{U L}$ for pions as a function of the Collins angle $\phi$. The curve for $\pi^{+}$corresponds to the fit $A=P_{1}+P_{2} \sin \left(\phi_{\pi}\right)$ with $P_{1}=-0.007 \pm 0.003$ and $P_{2}=0.020 \pm 0.004$. The corresponding curve for $\pi^{-}$ is given by $P_{1}=0.003 \pm 0.004$ and $P_{2}=-0.001 \pm 0.005$.

where the subscript $U L$ refers to unpolarized beam and longitudinally polarized target, provide a clear signature of the Collins effect. The data are shown in Fig. (9). For $\pi^{+}$the distribution shows a strong $\sin (\phi)$ dependence, the signature of the Collins effect, while the curve for $\pi^{-}$is isotropic. The difference between the $\pi^{+}$and $\pi^{-}$data can be ascribed to the dominance of $u$ quark fragmentation from the proton to the $\pi^{+}$.

Analysis of the data was performed under the assumption that the cross section factors into a hard scattering term times a fragmentation function. When this cross section is integrated over the final transverse momentum of the hadrons, the T-odd terms leading to single-spin asymmetries vanish. The various contributions to the $\phi$ dependent asymmetry are isolated by extracting moments of the cross section weighted by the corresponding $\phi$ dependent functions. In the present case, $\sin (\phi)$ was the weighing function of interest. Kotzinian and Mulders 39] have established that even with a longitudinally polarized target, one expects a significant single spin asymmetry with a $\sin (\phi)$ moment of the form

$$
\langle\sin (\phi)\rangle_{O L} \propto S_{T} \Sigma_{a} e_{a}^{2} h_{1}^{a}(x) H_{1}^{\perp a}+\ldots
$$

which arises from the small target spin component transverse to the direction of the virtual photon. Here, $H_{1}^{\perp a}$ is the Collins spin dependent fragmentation function. The analyzing powers for the beam and target polarizations, $A_{U L}^{\sin \phi}$ and $A_{L U}^{\sin \phi}$, were evaluated by calculating the azimuthially weighted moments of the event spectra. A substantial analyzing power is observed for $\pi^{+}, A_{U L}^{\sin \phi}=$ $0.022 \pm 0.005$ (stat.) \pm 0.003 (syst.), while for $\pi^{-}, A_{U L}^{\text {sin } \phi}=0.002 \pm 0.006$ (stat. $) \pm$ 


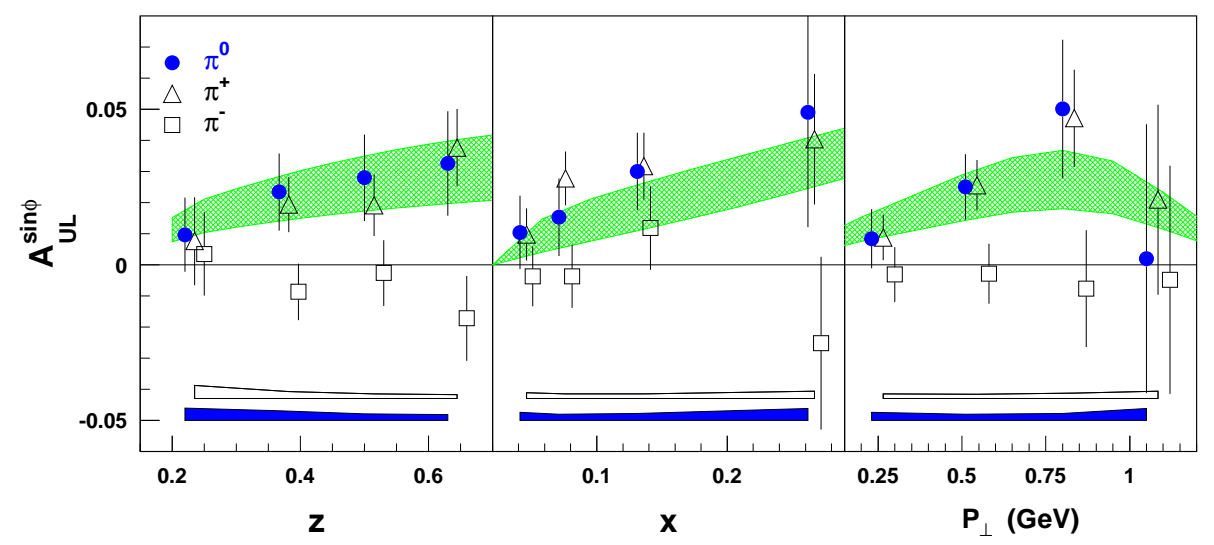

Figure 10: Analyzing power $A_{U L}^{s i n \phi}$ for pions as a function of the fractional pion energy $z$, the Bjorken variable $x$, and the pion transverse momentum $p_{\perp}$. Error bars include only statistical uncertainties. The open and filled bands at the bottom of the panels represent the systematic uncertainties for neutral and charged pions, respectively. The shaded areas show a range of model predictions, (see text).

0.003 (syst.). For both $\pi^{+}$and $\pi^{-}$the beam-related analyzing powers $A_{L U}^{s i n \phi}$ are consistent with zero, as is the other target related analyzing power $A_{U L}^{\sin 2 \phi}$. This is already a strong signal that pion single spin asymmetries provide a large analyzing power for measuring the transverse distribution function, $h_{1}\left(x, Q^{2}\right)$.

The measurements have been extended to include neutral pions. The results are summarized in Fig. (10) where $A_{U L}^{s i n \phi}$ is shown for each charge as a function of $z, x$, and the pion transverse momentum, $p_{\perp}$, after averaging over the other two kinematic variables. The $\pi^{0}$ and $\pi^{+}$analyzing powers exhibit similar behaviour in each variable. The increase of $A_{U L}^{s i n \phi}$ with increasing $x$ suggests that singlespin asymmetries are valence quark effects. The increase of $A_{U L}^{\sin \phi}$ with $p_{\perp}$ can be related to the dominant role of intrinsic quark transverse momentum when $p_{\perp}$ remains below about $1 \mathrm{GeV} / \mathrm{c}$. The results for $\pi^{+}$and $\pi^{0}$ follow the predictions of a model calculation 40 in which the distribution function of Eq. (13) is approximated by $h_{1}$. The range of predictions shown in Fig. (10) correspond to the limits $h_{1}=g_{1}$ (non-relativistic limit) and $h_{1}=\left(f_{1}+g_{1}\right) / 2$ (Soffer inequality), where $g_{1}$ and $f_{1}$ are the usual polarized and unpolarized distribution functions. The T-odd fragmentation function $H_{1}^{\perp(1)}(z)$ was assumed to follow the Collins parameterization[35. The success of this simple model calculation provides confidence that accurate determinations of transversity distributions are possible through observation of pion single spin asymmetries with transversely polarized targets. Such measurements are scheduled by the HERMES collaboration in the immediate future. 


\section{Deep Virtual Compton Scattering}

Deep virtual Compton scattering (DVCS) can be viewed as the scattering of a virtual photon generated in lepton scattering into the continuum. Although little data on the process are available, the process is of wide interest because exclusive DVCS is the simpliest example of a reaction described by a new class of parton distributions, i.e. generalizations of the usual forward parton distributions (pdf's). While ordinary pdf's give the probability of finding a quark with a momentum fraction $x=k / p$ in the nucleon, generalized parton distributions (GPD's) describe the removal of a quark $q\left(k-\frac{\Delta}{2}\right)$ and implantation of $q^{\prime}\left(k+\frac{\Delta}{2}\right)$. The example of DVCS is shown graphically in Fig. (11). Factorization theorms have been formulated 41 which demonstrate that contributions for hard exclusive reactions have the form of products of GPD's times hard scattering coefficients which are calculable from perturbative QCD. There are four families of GPD's, one pair of unpolarized distribution functions, $H^{q}(x, \xi, t)$ and $E^{q}(x, \xi, t)$, and a second pair of polarized distribution functions, $\tilde{H}^{q}(x, \xi, t)$ and $\tilde{E}^{q}(x, \xi, t)$. The relevant light cone variables are the longitudinal momentum fraction $x$, the skewness variable $\xi=-\Delta^{+} / 2 P^{+}$, and the four momentum transfer to the nucleon squared $t=\Delta^{2} . H^{q}$ and $\tilde{H}^{q}$ conserve the helicity of the nucleon, while $E^{q}$ and $\tilde{E}^{q}$ describe processes in which the nucleon helicity may flip. GPD's combine the character of ordinary parton distributions and nucleon form factors through their kinematic limits and moments, e.g. $q(x)=H^{q}(x, \xi=0, t=0)$, and $\Delta q(x)=\tilde{H}^{q}(x, \xi=0, t=0)$. The first moments constrain nucleon form factors through the relations $\int_{-1}^{1} d x H^{q}\left(x, \xi, \Delta^{2}\right)=F_{1}^{q}\left(\Delta^{2}\right), \int_{-1}^{1} d x E^{q}\left(x, \xi, \Delta^{2}\right)=$ $F_{2}^{q}\left(\Delta^{2}\right), \int_{-1}^{1} d x \tilde{H}^{q}\left(x, \xi, \Delta^{2}\right)=g_{A}^{q}\left(\Delta^{2}\right)$, and $\int_{-1}^{1} d x \tilde{E}^{q}\left(x, \xi, \Delta^{2}\right)=h_{A}^{q}\left(\Delta^{2}\right) . F_{1}(t)$ and $F_{2}(t)$ are the Dirac and Pauli form factors, while $g_{A}(t)$ and $h_{A}(t)$ are the axial vector and pseudoscalar form factors, respectively. The strong interest in GPD's comes, in part, from the observation 42 that their second moments can be connected to the total orbital angular momentum carried by the partons
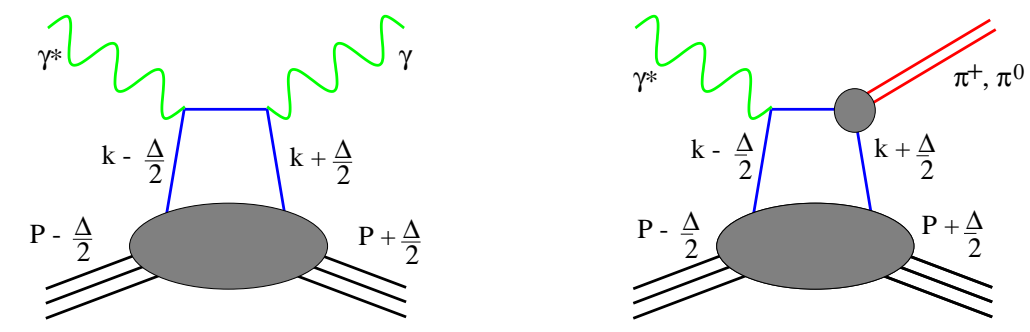

Figure 11: Graphic representation of processes involving generalized parton distributions, deep virtual Compton scattering (left) and exclusive pion production (right). The GPD describes the emission of a parton of momentum $k-\frac{\Delta}{2}$ and its reabsorption with momentum $k+\frac{\Delta}{2}$. 
through the relation

$$
J^{q}=\frac{1}{2} \Delta \Sigma+L^{q}=\frac{1}{2} \int_{-1}^{1} x d x\left[H^{q}(x, \xi, t=0)+E^{q}(x, \xi, t=0)\right] .
$$

Thus, GPD's may provide a measure of the $J^{q}$ and therefore with prior knowledge of $\Delta \Sigma$, of the orbital angular momentum of the partons, $L^{q}$.

DVCS is coherent with Bethe-Heitler bremstrahlung, and the interference between these two processes provides the opportunity to isolate DVCS amplitudes. The cross section for exclusive photon production is of the form 43

$$
\frac{d^{4} \sigma}{d \phi d t d Q^{2} d x}=\frac{x y^{2}}{32(2 \pi)^{4} Q^{4}} \frac{\left|\tau_{B H}+\tau_{D V C S}\right|^{2}}{\left(1+4 x^{2} m^{2} / Q^{2}\right)^{1 / 2}} .
$$

Both the magnitude and phase of the interference term can be measured with an unpolarized target. Measurements of the charge asymmetry with an unpolarized beam give

$$
d \Delta \sigma_{c h} \equiv d \sigma_{e^{+}}-d \sigma_{e^{-}} \sim \cos (\phi) \times \operatorname{Re}\left(\tau_{D V C S} \tau_{B H}\right),
$$

while measurements of the spin asymmetry with respect to the beam helicity give

$$
d \Delta \sigma_{L U} \equiv d \sigma_{\leftarrow}-d \sigma_{\rightarrow} \sim \sin (\phi) \times \operatorname{Im}\left(\tau_{D V C S} \tau_{B H}\right) .
$$

Thus, these asymmetries access the real and imaginary parts of the same interference amplitude which has the form

$$
\tau_{D V C S} \tau_{B H} \propto F_{1} \mathcal{H}_{1}+\frac{x_{B}}{2-x_{B}}\left(F_{1}+F_{2}\right) \tilde{\mathcal{H}}_{1}-\frac{\Delta_{2}}{4 M^{2}} F_{2} \mathcal{E}_{1} .
$$

The DVCS amplitudes of Eq. (18), $\mathcal{H}_{1}, \tilde{\mathcal{H}}_{1}$, and $\mathcal{E}_{1}$, can be given as convolutions 43 in $t$ of perturbative calculable hard scattering parts with GPD's for DVCS.

In HERMES DVCS has been measured with the HERA longitudinally polarized positron beam at DESY, with longitudinally polarized and unpolarized targets. Events were selected if they contained only one positron with a momentum larger than $3.5 \mathrm{GeV}$, and only one photon with an energy greater that $0.8 \mathrm{GeV}$ in the calorimeter. Kinematic cuts of $Q^{2}>1 \mathrm{GeV}^{2}, W^{2}>4 \mathrm{GeV}^{2}$, and $\nu<24 \mathrm{GeV}$ were imposed. In addition, the cut $15<\Theta_{\gamma * \gamma}<70$ mrad was used to avoid false asymmetries from bias in reconstruction of small angles. A contamination of events from $\pi^{0} \rightarrow 2 \gamma$ decay which arises from the calorimeter granularity was determined to be $<5 \%$. The missing mass spectrum for DVCS is shown in Fig. (12), where it is compared to results of a Monte Carlo simulation which includes photons generated from DIS as well as those resulting from the exclusive Bethe-Heitler process, $e+p \rightarrow e^{\prime}+p+\gamma$. Because of the finite resolution of the spectrometer, $M_{x}^{2}$ may be negative, in which case by definition, $M_{x}=-\sqrt{-M_{x}^{2}}$. The missing mass region between -1.5 and $+1.7 G e V$ was selected for isolating exclusive DVCS. This region contains both exclusive scattering to the proton ground state and the $\Delta(1232)$ resonance. However, because the Bethe-Heitler process is strongly dominated by the elastic channel the 

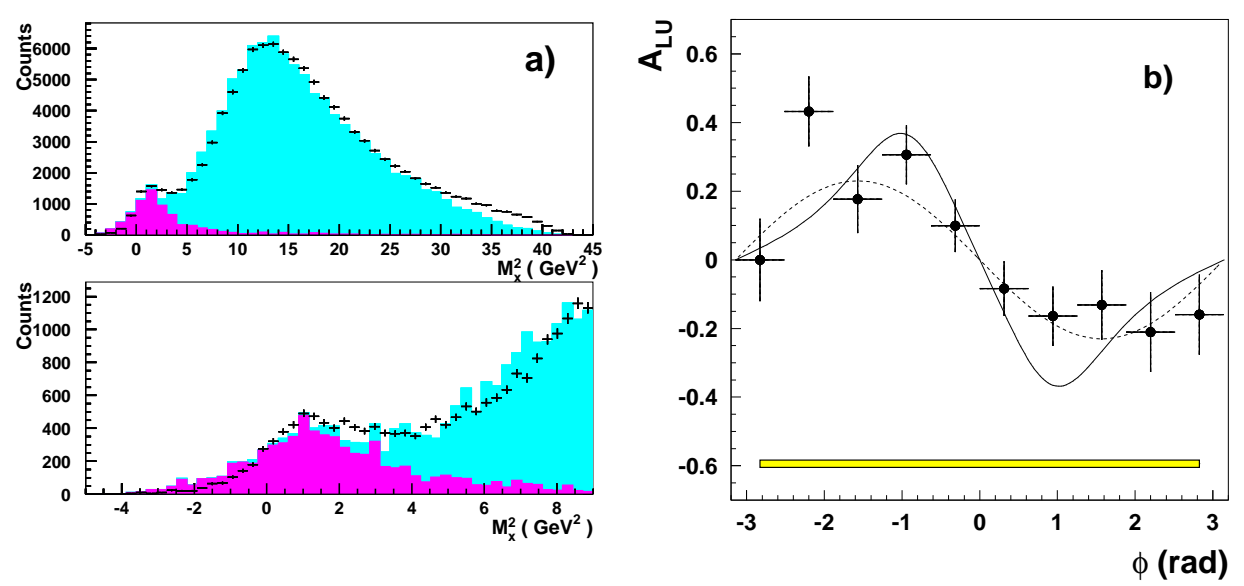

Figure 12: a) The measured distribution of photons observed in hard electroproduction as a function of the missing mass squared $M_{x}^{2}$. The upper panel shows the full kinematic range, while the low $M_{x}^{2}$ domain is presented in the lower panel. The light-gray histogram represents the results of a Monte Carlo simulation of the experiment which includes fragmentation processes and the BetheHeitler process, while the dark-shaded histogram represents only the BetheHeitler contribution. b) Beam-helicity analyzing power $A_{L U}$ as a function of the azimuthal angle $\phi$. The dashed curve represents a $\sin \phi$ dependence with an amplitude of -0.23 , while the solid curve is a theoretical calculation in the approximation of small $t$ (see text).

spin asymmetries are expected to have a small contribution from the $\Delta(1232)$ resonance.

The azimuthal distribution of events in the missing mass window centered on the proton mass is presented in Fig. (12) where the beam-helicity analyzing power

$$
A_{L U}(\Theta)=\frac{1}{\left\langle\left|P_{b}\right|\right\rangle} \frac{N^{+}(\phi)-N^{-}(\phi)}{N^{+}(\phi)+N^{-}(\phi)}
$$

is plotted. The data show the characteristic $\sin \phi$ dependence expected from Eq. (17). The solid curve of Fig. (12) is the result of a calculation 44 with GPD's in the approximation of small $t$ where factorization into contributions from form factors and parton distributions is reasonable. The calculation gives a good description of the data. Checks on the beam-helicity and missing mass dependence were performed by examining the distributions of the $\sin \phi$-weighted moments which were defined as

$$
A_{L U}^{s i n \phi^{ \pm}}=\frac{2}{N^{ \pm}} \sum_{i=1}^{N^{ \pm}} \frac{\sin \phi_{i}}{\left|P_{b}\right|_{i}} .
$$



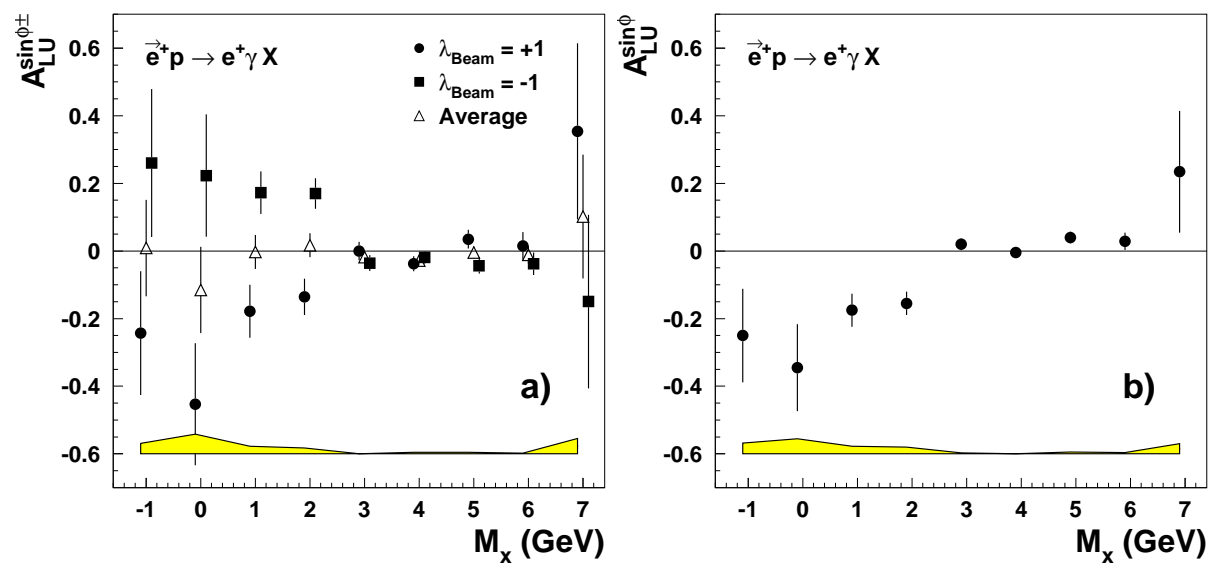

Figure 13: a) The $\sin \phi$-moment $A_{L U}^{\sin \phi^{ \pm}}$as a function of the missing mass for positive beam helicity (circles), negative beam-helicity (squares) and the averaged helicity (open triangles). b) The beam-helicity analyzing power $A_{L U}^{\sin \phi}$ as a function of the missing mass.

The $\sin \phi$ moments are shown in Fig. (13) for the two beam helicities together with their average. As expected, the sign of the moment is opposite for the two helicities and their average is consistent with zero. Also, as expected the moments vanish for high missing mass. The data for the two beam helicities can be combined to determine the beam-helicity analyzing power

$$
A_{L U}^{s i n \phi}=\frac{2}{N} \sum_{i=1}^{N} \frac{\sin \phi_{i}}{\left(P_{b}\right)_{i}}
$$

Combining the data in the $M_{x}$ region between -1.5 and $+1.7 \mathrm{GeV}$ yields the result $A_{L U}^{\sin \phi}=-0.23 \pm 0.04$ (stat.) \pm 0.03 (system) which is to be compared with the theoretical calculation 44 of $A_{L U}^{s i n \phi}=-0.37$. The average values of the kinematic variables corresponding to this measurement are $\langle x\rangle=0.11$, $<Q^{2}>=2.6 \mathrm{GeV}^{2}$, and $\left\langle t>=0.27 \mathrm{GeV}^{2}\right.$. The HERMES results provide a clear demonstration of a strong experimental signature for DVCS which can be exploited to study GPD's in what may be the conceptually clearest case. The measurement described here is the initial step in a program of systematic studies, but the results conform to theoretical expectations and foretell a new rich area of study.

\section{The Future}

In spite of many years of experiments, nucleon spin structure remains a complex and subtle problem. A detailed decomposition of the spin of the nucleon 
remains elusive. However, as the data from the HERMES experiment indicate, we are beginning to obtain information on some of the central questions. What is the polarization of the gluons and the strange sea? How are transverse and longitudinal spin distributions related? Future measurements at HERMES will focus on direct measurements of transversity with transversely polarized targets. Measurements planned in the RHIC spin program 45, 46] will provide the first detailed probing of gluon spin distributions. Measurements of DVCS may provide sufficient access to GPD's to enable mapping out their variation with kinematic variables $(x, \xi, t)$, posing a major challenge to experimenters. Will accurate determination of the second moments of the GPD's be possible? Can we access the parton angular momenta $L_{q}$ with measurements of DVCS?

\section{Acknowledgements}

The support of the DESY management and staff and the staffs of the collaborating institutions is gratefully acknowledged. The author wishes to thank R. J. Holt, G. van der Steenhoven, and P. Reimer for a careful reading of the manuscript. The author also acknowledges the massive efforts of all the HERMES collaborators which have made the program a success. This work was supported in part by the U.S. Department of Energy and National Science Foundation.

\section{References}

[1] M. J. Alguard et al., Phys. Rev. Lett. 37, 1261 (1976); 41, 70 (1978); G. Baum et al., ibid. 51, 1135 (1983).

[2] R. L. Jaffe and A. Manohar, Nucl. Phys. B337, 509 (1990).

[3] J. Ashman et al., Phys. Lett. 206, 364 (1988); Nucl. Phys. B328, 1 (1989).

[4] G. Altarelli, R. D. Ball, S. Forte, and G. Ridolfi, Nucl.Phys. B496, 337 (1997).

[5] R. Mertig and W. L. van Neerven, Zeit. Phys. C70, 637 (1996); W. Vogelsang, Phys. Rev. D54, 2023 (1996).

[6] B. Adeva et al., Phys. Rev. D58, 112002 (1998).

[7] D. Adams et al., Phys. Rev. D56, 5330 (1997).

[8] E. Leader, A. V. Sidorov, and D. B. Stamenov, Phys. Lett. B488, 283 (2000).

[9] J. Stewart, Proc. of the Workshop on Polarised Gas Targets and Polarised Beams, ed. R. J. Holt and M. A. Miller, AIP Conf. Proc. 421, 69 (1997).

[10] A. A. Sokolov and I. M. Ternov, Sov. Phys. Doklady 8, 12031964. 
[11] D. P. Barber et al., Phys. Lett. B343, 435 (1997).

[12] M. Beckmann et al., Nucl. Instr. Meth. A479, 334 (2002).

[13] C. Baumgarten et al., Nucl. Instr. Meth. A, in press.

[14] M. C. Simani et al., submitted to Nucl. Instr. Meth. A.

[15] Th. Benisch et al., Nucl. Instr. Meth. A471, 314 (2001).

[16] K. Ackerstaff et al., Nucl. Instr. Meth. A417, 230 (1998).

[17] N. Akopov et al., Nucl. Instr. Meth. A479, 511 (2002).

[18] A. Airapetian et al., Phys. Lett. B442, 484 (1998).

[19] M. A.Funk, PhD thesis, Univ. Hamburg, ISSN 1435-8085, 1998

[20] J. M. Niczyporuk and E. E. W. Bruins, Phys. Rev. D58, 091501 (1998).

[21] K. Ackerstaff et al., Phys. Lett. B464, 123 (1999).

[22] B. Adeva et al., Phys. Lett. B369, 93 (1996).

[23] H. L. Lai et al., Eur. Phys. J. C12, 375 (2000).

[24] G. Ingelman, A. Edin, and J. Rathsman, DESY Report 96-057, (1996).

[25] M. Gluck, E. Reya, and A. Vogt, Eur. Phys. J. C5, 461 (1998).

[26] K. Ackerstaff et al., Phys. Lett. B464, 123 (1999).

[27] B. Adeva et al., Phys. Lett. B420, 180 (1998).

[28] B. Adeva et al., Phys. Lett. B320, 400 (1994).

[29] B. Dressler et al., Eur. Phys. J. C14, 147 (2000).

[30] Alessendro Bravar, Dietrich von Harrach, and Aram Kotzinian, Phys. Lett. B349, 349 (1998).

[31] A. Airapetian et al., Phys. Rev. Lett. 84, 2584 (2000).

[32] M. Fantannaz, D. Schiff, and B. Pire, Z. Phys. C8, 349 (1981).

[33] T. Gehrmann and W. J. Stirling, Phys. Rev. D53, 6100 (1996).

[34] M. Glück, E. Reya, M Stratmann, and W. Vogelsang, Phys. Rev. D53, 4775 (1996).

[35] J. Collins, Nucl. Phys. B396, 161 (1993).

[36] X. Artu, J. Czyzewski, and H. Yabuki, Z. Phys. C73, 527 (1997). 
[37] D. L. Adams et al., Phys. Lett. B264, 462 (1991); D. L. Adams et al., Z. Phys. C56, 181 (1992).

[38] P. J. Mulders and R. D. Tangerman, Nucl. Phys. B461, 197 (1996).

[39] A. M. Kotzinian and P. J. Mulders, Phys. Lett. B406, 373 (1997).

[40] E. De Sanctis, W. D. Nowak, and K. A. Oganessyan, Phys. Lett. B483, 69 (2000).

[41] J. C. Collins, L. Frankfurt, and M. Strikman, Phys. Rev. D56, 2982 (1997).

[42] X. Ji, Phys. Rev. Lett. 78, 610 (1997), Phys. Rev. D55, 7114 (1997).

[43] A. V. Belitsky et al., Nucl. Phys. B593, 289 (2001).

[44] N. Kivel, M. V. Polyakov, and M. Vanderhaegen, Phys. Rev. D63, 114014 (2001).

[45] N. Saito, Nucl. Phys. A638, 575c (1998).

[46] S. E. Vigdor, SPIN98 Proceedings (World Scientific, Singapore, 1998), 151. 